
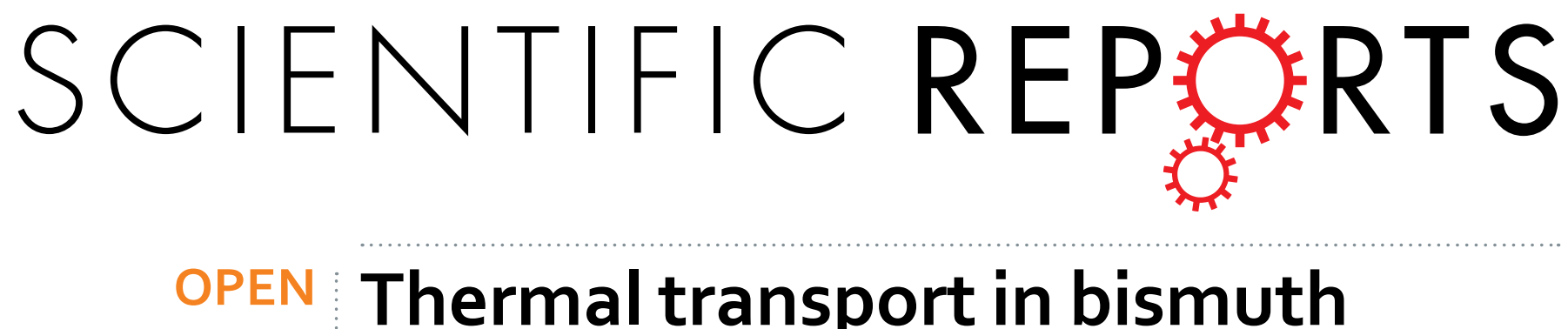

\title{
Thermal transport in bismuth telluride quintuple layer: mode- resolved phonon properties and substrate effects
}

Accepted: 19 May 2016

Published: 06 June 2016

\section{Cheng Shao \& Hua Bao}

The successful exfoliation of atomically-thin bismuth telluride $\left(\mathrm{Bi}_{2} \mathrm{Te}_{3}\right)$ quintuple layer $(\mathrm{QL})$ attracts tremendous research interest in this strongly anharmonic quasi-two-dimensional material. The thermal transport properties of this material are not well understood, especially the mode-wise properties and when it is coupled with a substrate. In this work, we have performed molecular dynamics simulations and normal mode analysis to study the mode-resolved thermal transport in freestanding and supported $\mathrm{Bi}_{2} \mathrm{Te}_{3} \mathrm{QL}$. The detailed mode-wise phonon properties are calculated and the accumulated thermal conductivities with respect to phonon mean free path (MFP) are constructed. It is shown that $60 \%$ of the thermal transport is contributed by phonons with MFP longer than $20 \mathrm{~nm}$. Coupling with $a$ $\mathrm{SiO}_{2}$ substrate leads to about $60 \%$ reduction of thermal conductivity. Through varying the interfacial coupling strength and the atomic mass of substrate, we also find that phonon in $\mathrm{Bi}_{2} \mathrm{Te}_{3} \mathrm{QL}$ is more strongly scattered by interfacial potential and its transport process is less affected by the dynamics of substrate. Our study provides an in-depth understanding of heat transport in $\mathrm{Bi}_{2} \mathrm{Te}_{3} \mathrm{QL}$ and is helpful in further tailoring its thermal property through nanostructuring.

Bismuth telluride as one of the best room temperature thermoelectric materials has attracted significant attention recently ${ }^{1-5}$. The performance of thermoelectric materials is characterized by its figure of merit

$$
Z T=\frac{S^{2} \sigma T}{\kappa_{e}+\kappa_{l}},
$$

where $S, \sigma, T, \kappa_{e}$, and $\kappa_{l}$ are the Seebeck coefficient, electrical conductivity, absolute temperature, electrical thermal conductivity, and lattice thermal conductivity, respectively. One effective approach to enhance $Z T$ is to reduce the lattice thermal conductivity of a thermoelectric material ${ }^{1-3}$. Significant progress has been made in the last decade to decrease the lattice thermal conductivity of $\mathrm{Bi}_{2} \mathrm{Te}_{3}$-based materials. For example, in nanostructured bismuth antimony telluride bulk alloy, a peak $Z T$ of 1.4 can be achieved at $100^{\circ} \mathrm{C}^{1}$. The $Z T$ value of p-type $\mathrm{Bi}_{2} \mathrm{Te}_{3} /$ $\mathrm{Sb}_{2} \mathrm{Te}_{3}$ superlattice was reported to be as high as 2.4 at room temperature, which is mainly due to the controlled transport of phonons and electrons in the superlattices ${ }^{2}$. Comparing to nanostructured bulk materials, low dimensional materials have large surface-to-volume ratios and size effects, which could be utilized to further enhance the $Z T$ value ${ }^{6-8}$. A few quintuple layers ( $\left.\mathrm{QLs}\right) \mathrm{Bi}_{2} \mathrm{Te}_{3}$ recently attracted great interest on its thermoelectric property due to its quasi-two-dimensional (2D) structure ${ }^{9}$. For example, Zhang et al. studied the electron and phonon transport properties in $\mathrm{Bi}_{2} \mathrm{Te}_{3} \mathrm{QL}$ and found that the $Z T$ value can be optimized to as high as 2.2 at $800 \mathrm{~K}^{10}$. Qiu and Ruan investigated the thermal transport in few-QLs $\mathrm{Bi}_{2} \mathrm{Te}_{3}$ thin films using molecular dynamics (MD) simulations ${ }^{11}$. They found that the thermal conductivity of $\mathrm{Bi}_{2} \mathrm{Te}_{3} \mathrm{QL}$ first decreases and then increases with the number of QLs, which is attributed to the interplay between Umklapp scattering and boundary scattering ${ }^{11}$. It is also shown that the thermal conductivity of QLs can be reduced by introducing nanopores ${ }^{11}$. More recently, Park et al. calculated the thermal conductivity of $\mathrm{Bi}_{2} \mathrm{Te}_{3}$ thin films by solving the Boltzmann transport equation under the relaxation time approximation ${ }^{12}$. They extended the Klemens-Callaway's model to incorporate the phonon scattering process due to the van der Waals force at the interface of adjacent $\mathrm{Bi}_{2} \mathrm{Te}_{3}$ QLs and could correctly 


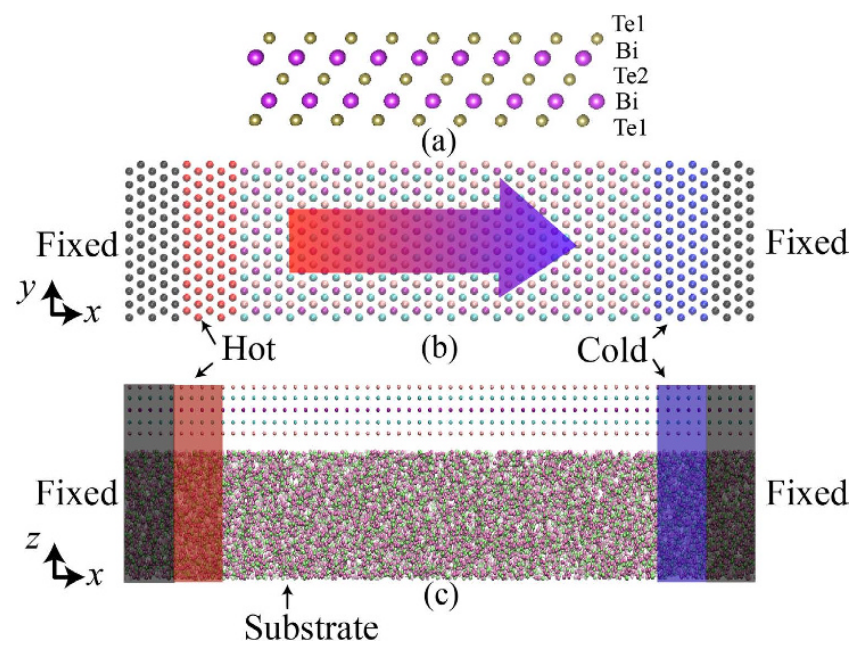

Figure 1. (a) Atomic structure (side view) of $\mathrm{Bi}_{2} \mathrm{Te}_{3} \mathrm{QL}$. (b) NEMD simulation model (top view) for freestanding $\mathrm{Bi}_{2} \mathrm{Te}_{3}$. (c) NEMD simulation model (side view) for supported $\mathrm{Bi}_{2} \mathrm{Te}_{3}$.

reproduce the anisotropic heat transport of $\mathrm{Bi}_{2} \mathrm{Te}_{3}{ }^{12}$. While these studies provided a better understanding of electronic and thermal transport properties in $\mathrm{Bi}_{2} \mathrm{Te}_{3} \mathrm{QL}$, detailed information about mode-resolved phonon transport properties and its contribution to thermal conductivity is still missing. Such information is crucial for a better understanding of thermal transport and tailoring the thermal conductivity ${ }^{13,14}$.

On the other hand, in the experimental characterization of thermal properties, the $2 \mathrm{D}_{2} \mathrm{Te}_{3}$ QLs could be supported on a substrate ${ }^{15,16}$. More generally, the coupling with another material (typically unavoidable) could affect the thermal transport of low-dimensional materials ${ }^{5,17}$. There have been quite a few studies focusing on the thermal transport of low-dimensional materials when it is weakly bonded to another material (typically to a substrate $)^{18-21}$. It is generally believed that the properties of low dimensional materials are very sensitive to the presence of substrate ${ }^{18-21}$. For example, Ong et al. studied the substrate effects on thermal transport in graphene and found that coupling to the substrate can reduce the thermal conductivity of graphene by an order of magnitude, but further increasing the coupling strength will enhance the thermal transport, due to the coupling of flexural acoustic phonons with substrate Rayleigh waves ${ }^{19}$. Qiu and Ruan performed a phonon spectral analysis to study the effect of silicon dioxide substrate on graphene ${ }^{22}$. They found that the presence of silicon dioxide substrate can reduce the phonon relaxation times (RTs) on all phonon branches ${ }^{22}$. There are also some studies showing that the thermal conductivity of could be enhanced due to the coupling with another material. For example, Zhang et al. studied the effect of substrate on heat transport in two-dimensional (2D) silicene and found that depending on the different crystal types of silicon carbide substrate, the thermal conductivity of 2D silicene could be either enhanced or suppressed ${ }^{23}$. Guo et al. investigated thermal transport in two coupled Fermi-Pasta-Ulam chains. The results show that in certain regions the coupling to another chain can increase the thermal conductivity of the chain $^{20}$. They believe that the enhancement of thermal conductivity is due to the reduction of anharmonic phonon scattering, which is induced by the shift of the phonon band to the low wave vector ${ }^{20}$. $\mathrm{Bi}_{2} \mathrm{Te}_{3} \mathrm{QL}$ is different from those materials in previous studies, because it has strong anharmonicity and extremely low thermal conductivity. The detailed effects of substrate on its thermal transport remain unclear and a systematic investigation is highly desirable.

In this work, we adopt MD based methods to study the phonon transport in $2 \mathrm{D} \mathrm{Bi}_{2} \mathrm{Te}_{3} \mathrm{QL}$. Thermal conductivities of freestanding and supported $\mathrm{Bi}_{2} \mathrm{Te}_{3} \mathrm{QL}$ are first calculated through non-equilibrium molecular dynamics (NEMD) and Green-Kubo methods. Then the normal mode analysis (NMA) method is employed to obtain the mode-resolved phonon transport properties in $\mathrm{Bi}_{2} \mathrm{Te}_{3}$ QL. The structure of $\mathrm{Bi}_{2} \mathrm{Te}_{3}$ QL coupled with an $a-\mathrm{SiO}_{2}$ substrate is also investigated to explore the substrate effects. Accumulated thermal conductivities with respect to MFP for both freestanding and supported $\mathrm{Bi}_{2} \mathrm{Te}_{3}$ are constructed to quantify the contribution of different phonon modes to thermal conductivity. We also investigated the effect of coupling strength and the atomic mass of substrate on the heat transfer in $\mathrm{Bi}_{2} \mathrm{Te}_{3} \mathrm{QL}$.

\section{Methods}

Molecular dynamics simulations. In this work, MD based methods are adopted to study the thermal transport properties of $\mathrm{Bi}_{2} \mathrm{Te}_{3}$ QL. LAMMPS package is used to carry out all the MD simulations ${ }^{24}$. It should be noted that there are also some recent works using the first-principles methods to study the thermal properties in $\mathrm{Bi}_{2} \mathrm{Te}_{3}{ }^{10,25}$. Although first-principles simulation can extract more accurate force constants, it is still difficult to incorporate higher order anharmonicity ${ }^{26,27}$. The large computational cost also made it is difficult to be applied to study a $\mathrm{Bi}_{2} \mathrm{Te}_{3} \mathrm{QL}$ or the effect of the external perturbation from a substrate.

The atomic structure of $\mathrm{Bi}_{2} \mathrm{Te}_{3} \mathrm{QL}$ is shown in Fig. 1(a). It contains five atomic layers with a sequence of $\mathrm{Te} 1-\mathrm{Bi}-\mathrm{Te} 2-\mathrm{Bi}-\mathrm{Te}$. Each layer has a hexagonal crystal structure with lattice constant $a=4.369 \AA$. The thickness of the $\mathrm{QL}$ is $10.14 \AA$, which is one-third of lattice constant of bulk $\mathrm{Bi}_{2} \mathrm{Te}_{3}$ along the cross-plane direction. A two-body Morse-type potential developed by Qiu et al. is used to model the interaction between atoms in $\mathrm{Bi}_{2} \mathrm{Te}_{3} \mathrm{QL}^{28}$. An $a-\mathrm{SiO}_{2}$ substrate is used to study the effect of substrate on heat transport in $\mathrm{Bi}_{2} \mathrm{Te}_{3} \mathrm{QL}$. We choose $a$-SiO ${ }_{2}$ for its 
widely used in experiments ${ }^{15}$. A Tersoff-type potential parameterized by Munetoh et al. ${ }^{29}$ is used to model the interaction between $\mathrm{Si}$ and $\mathrm{O}$ atoms. To generate the $a-\mathrm{SiO}_{2}$, we first heat the $\beta$-cristobalite to $6000 \mathrm{~K}$ using a Langevin thermostat and maintain that temperature for $10 \mathrm{ps}$. Then the system is slowly quenched to $300 \mathrm{~K}$ at a rate of $10^{12} \mathrm{~K} / \mathrm{s}^{30}$. The $a-\mathrm{SiO}_{2}$ substrate is $2 \mathrm{~nm}$ thick, which should be thick enough to eliminate the size effect based on the previous studies ${ }^{22,31,32}$. Because of the van der Waals nature of interaction between the $\mathrm{Bi}_{2} \mathrm{Te}_{3} \mathrm{QL}$ and substrate, a Lennard-Jones ( $\mathrm{LJ})$ potential is adopted to describe the interaction, which has the form of

$$
U_{i j}=4 \varepsilon_{i j}\left[\left(\frac{\sigma_{i j}}{r_{i j}}\right)^{12}-\left(\frac{\sigma_{i j}}{r_{i j}}\right)^{6}\right],
$$

where $\varepsilon_{i j}$ is the potential well depth, $\sigma_{i j}$ is the distance at which the potential energy reaches zero, and $r_{i j}$ is the separation between atoms $i$ and $j$. In this study, the parameters are obtained based on the universal force model ${ }^{33}$, which gives $\varepsilon_{\mathrm{Bi}-\mathrm{Si}}=17.9 \mathrm{meV}, \sigma_{\mathrm{Bi}-\mathrm{Si}}=3.9 \AA, \varepsilon_{\mathrm{Bi}-\mathrm{O}}=7.6 \mathrm{meV}, \sigma_{\mathrm{Bi}-\mathrm{O}}=3.5 \AA, \varepsilon_{\mathrm{Te}-\mathrm{Si}}=17.3 \mathrm{meV}, \sigma_{\mathrm{Te}-\mathrm{Si}}=3.9 \AA$, $\varepsilon_{\mathrm{Te}-\mathrm{O}}=6.7 \mathrm{meV}$, and $\sigma_{\mathrm{Te}-\mathrm{O}}=3.5 \AA$. The cutoff distance for LJ potential is set to be $10 \AA$.

We use both NEMD and Green-Kubo methods to calculate the thermal conductivity of freestanding $\mathrm{Bi}_{2} \mathrm{Te}_{3}$ $\mathrm{QL}$, and use NEMD to calculate the thermal conductivity of supported $\mathrm{Bi}_{2} \mathrm{Te}_{3}$ QL. The schematic of the simulation domain used in NEMD is shown in Fig. 1(b,c). The outermost regions are static regions in which the atoms are fixed during the NEMD process. Next to the fixed regions are the hot and cold thermal reservoirs. Periodic boundary condition is applied to all three directions, while a vacuum layer of $5 \mathrm{~nm}$ is used in $z$ direction (cross plane direction) to model the freestanding situation. The width ( $y$ direction) of the simulation domain is set to be $6 \mathrm{~nm}$ after convergence tests. It is well known that NEMD methods suffer from the finite size effect in predicting the thermal conductivity. In order to eliminate this effect, we vary the domain size along the $x$ direction and use the extrapolation method to obtain the thermal conductivity. In freestanding cases, we vary the length from 16 to $80 \mathrm{~nm}$. For the supported cases, due to the large computational costs, the maximum length along the heat flux direction is $40 \mathrm{~nm}$. A time step of $2 \mathrm{fs}$ is used the simulations of freestanding $\mathrm{Bi}_{2} \mathrm{Te}_{3} \mathrm{QL}$. Due to the existence high of frequency vibration modes in $a-\mathrm{SiO}_{2}$ substrate, we use a time step of $0.25 \mathrm{fs}$ for supported $\mathrm{Bi}_{2} \mathrm{Te}_{3} \mathrm{QL}$. The system is first equilibrated in an NVT ensemble at $300 \mathrm{~K}$ for $0.5 \mathrm{~ns}$, and then switched to NPT ensemble to release the internal stress for $1 \mathrm{~ns}$. After that atoms at two ends are fixed and the temperature of two reservoirs are maintained at $320 \mathrm{~K}$ and $280 \mathrm{~K}$ respectively through the velocity rescaling method ${ }^{34}$. After obtaining the steady state temperature gradient and heat flux across the system, Fourier's law is applied to calculate the thermal conductivity.

For the Green-Kubo method, the thermal conductivity is obtained from the autocorrelation of the heat current vector. Equilibrium molecular dynamic (EMD) simulations are carried out to calculate heat current vectors. The simulation domain used for EMD simulations is $5.6 \times 5.2 \mathrm{~nm}^{2}$ in the $x$ and $y$ direction (containing 910 atoms), while in $z$ direction a length of $10 \mathrm{~nm}$ is used to model the freestanding structure. According to the previous $\mathrm{MD}$ study on $\mathrm{Bi}_{2} \mathrm{Te}_{3} \mathrm{QL}^{11}$, our simulation domain size is large enough to obtain converged thermal conductivity. We first run the pre-equilibrium procedures similar to the NEMD simulation to make sure the system reaches equilibrium state. After that the system is switched to an NVE ensemble for $2 \mathrm{~ns}$ and the heat current data is recorded every $10 \mathrm{fs}$. These heat current data are then used to calculate the heat current autocorrelation function (HCACF). Fifteen independent simulations (different in initial velocities) are used to explore the phase space and make sure our results are true ensemble average $\mathrm{e}^{30,35}$. Here a direct integral method is used to integrate the HCACF and to obtain the thermal conductivity. It should be noted that in some previous works, a low-pass filter is used to remove the optical component in the HCACF and the exponential fitting method is used to extract the final thermal conductivity ${ }^{28,36}$. To make a comparison, we used the directed integral method to integrate the HCACF with and without a low-pass filter (the cutoff frequency is $0.5 \mathrm{THz}$ ) and found they yield similar results.

Normal mode analysis. While NEMD and Green-Kubo method can provide an overall thermal conductivity, no information at the phonon level can be obtained. In order to obtain better insights about heat transport in $\mathrm{Bi}_{2} \mathrm{Te}_{3} \mathrm{QL}$, a mode-wise analysis is necessary. In normal mode analysis (NMA), the trajectories of each atom are projected onto the normal modes and the amplitude of each normal mode can be written as ${ }^{37,38}$

$$
Q(\mathbf{k}, \nu, t)=\frac{1}{N^{1 / 2}} \sum_{j l} m_{j}^{1 / 2} \exp \left(-\mathrm{ik} \cdot r_{j l}\right) \mathrm{e}_{j}^{*}(\mathbf{k}, \nu) \cdot u_{j l}(t)
$$

where $Q(\mathbf{k}, \nu, t)$ denotes the amplitude of a normal-mode with wave vector $\mathbf{k}$ and polarization $\nu$ at time $t, N$ is the number of unit cells in the simulation domain, $m$ is the mass, $r$ is the equilibrium position of an atom, e $(\mathbf{k}, \nu)$ is the mode eigenvector, and $u$ is the displacement. The subscript $j$ denotes the $j$-th atom in a unit cell, $l$ denotes the $l$-th unit cell and the superscript *denotes the complex conjugate.

When the lattice is purely harmonic, the normal-mode amplitudes will be periodic functions of time. The anharmonic forces cause the damping of the normal-mode. If the anharmonic effect is small, the normal-mode amplitudes can be written as ${ }^{39}$

$$
Q(\mathbf{k}, \nu, t)=Q(\mathbf{k}, \nu, 0) e^{-i\left[\omega_{0}(\mathbf{k}, \nu)+\Delta(\mathbf{k}, \nu)-i \Gamma(\mathbf{k}, \nu)\right] t},
$$

where $\omega_{0}(\mathbf{k}, \nu)$ is the unperturbed harmonic frequency of this mode, $\Delta(\mathbf{k}, \nu)$ is the frequency shift, and $\Gamma(\mathbf{k}, \nu)$ is the linewidth. The phonon spectral linewidth $\Gamma(\mathbf{k}, \nu)$ and the phonon relaxation time $\tau(\mathbf{k}, \nu)$ have the following relationship 


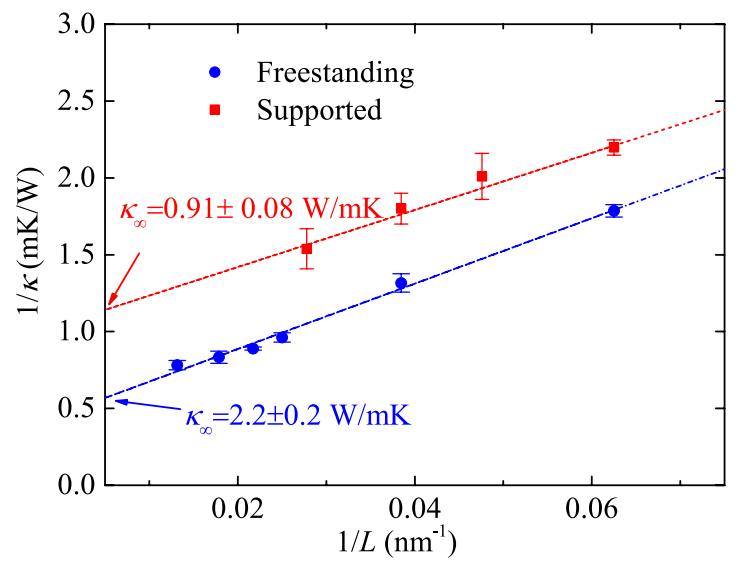

Figure 2. Thermal conductivity of freestanding and supported $\mathrm{Bi}_{2} \mathrm{Te}_{3} \mathrm{QL}$ calculated through NEMD methods and the extrapolation value at $L=\infty$.

$$
\tau(\mathbf{k}, \nu)=\frac{1}{2 \Gamma(\mathbf{k}, \nu)} .
$$

In order to obtain the phonon relaxation time, we apply a Fourier transform to the time derivative of each normal-mode and express the spectral energy density (SED) of this mode as:

$$
\mathrm{F}(\dot{Q}(\mathbf{k}, \nu, t))^{2}=\Phi(\mathbf{k}, \nu, \omega)=\frac{c(\mathbf{k}, \nu)}{[2 \tau(\mathbf{k}, \nu)(\omega-\omega(\mathbf{k}, \nu))]^{2}+1},
$$

where F denotes the Fourier transform, $Q(\mathbf{k}, \nu, t)$ is the time derivative of $Q(\mathbf{k}, \nu, t), \omega(\mathbf{k}, \nu)$ is the anharmonic phonon frequency (equals to $\omega_{0}(\mathbf{k}, \nu)+\Delta(\mathbf{k}, \nu)$ from Equation (4)), and $\Phi(\mathbf{k}, \nu, \omega)$ is the SED of this mode and can be expressed as a Lorentzian function. The phonon frequency and relaxation time are obtained by fitting the SED plot with a Lorentzian function. The location of peak gives the phonon frequency and the inverse of full width at half maximum $2 \Gamma$ is the phonon relaxation time $\tau$.

Similar to previous studies, we make the isotropic approximation for the first Brillouin zone $\mathrm{e}^{32,40}$. A simulation domain of $13.1 \times 6.1 \mathrm{~nm}^{2}$ in the $x$ and $y$ direction is used in our study. It should be noted that available k points are depending on the size of simulation domain ${ }^{41}$, so there are $21 \mathrm{k}$ points available along the $\Gamma$ to $\mathrm{K}$ direction in our simulations. The lattice dynamics program GULP is used to calculate the eigenvectors ${ }^{42}$. The atomic positions and velocities are recorded at a $10 \mathrm{fs}$ interval during an NVE process at $300 \mathrm{~K}$. A trajectory of $20 \mathrm{~ns}$ is used in the NMA to extract the phonon frequencies and relaxation times. We also apply the NMA to bulk $\mathrm{Bi}_{2} \mathrm{Te}_{3}$. In bulk $\mathrm{Bi}_{2} \mathrm{Te}_{3}$ the lattice constant along the cross-plane direction is $30.42 \AA$, which contains three QLs. Periodic boundary condition is used in the cross-plane direction to model the bulk situation. All the other simulation details are similar to that used for $\mathrm{Bi}_{2} \mathrm{Te}_{3} \mathrm{QL}$.

\section{Results and Discussions}

Thermal conductivity of freestanding and supported $\mathrm{Bi}_{2} \mathrm{Te}_{3} \mathrm{QL}$. Thermal conductivity values of freestanding and supported $\mathrm{Bi}_{2} \mathrm{Te}_{3} \mathrm{QL}$ at different lengths calculated from the NEMD method are shown in Fig. 2. These data points were extrapolated with a linear function to obtain the thermal conductivity at infinite length ${ }^{43}$, which are $2.2 \pm 0.2 \mathrm{~W} / \mathrm{mK}$ and $0.91 \pm 0.08 \mathrm{~W} / \mathrm{mK}$ for freestanding and supported cases, respectively. For the freestanding case, there seems to be an onset of a convergence of the thermal conductivity at the point when $L=80 \mathrm{~nm}\left(1 / L=0.0125 \mathrm{~nm}^{-1}\right)$. Adopting linear fitting may overestimate the thermal conductivity. Therefore, we also tried to extrapolate thermal conductivity using a quadratic fitting, and it yields $2.1 \mathrm{~W} / \mathrm{mK}$. The Green-Kubo method was also used to calculate the in-plane thermal conductivity of freestanding $\mathrm{Bi}_{2} \mathrm{Te}_{3} \mathrm{QL}$ for comparison. To obtain the thermal conductivity, one can either fit the HCACF with exponential function, or directly integrate the HCACF. In a previous work by Qiu and Ruan ${ }^{28}$, the former approach was adopted and a low-pass filter was used to remove the high frequency oscillation. In our simulation the direct integration method is used. From Fig. 3, one can see that whether removing the high frequency oscillation components of the HCACF does not obviously affect the final thermal conductivity value. The result is $1.8 \pm 0.1 \mathrm{~W} / \mathrm{mK}$, which is consistent with our NEMD result and agrees well with the work by Qiu and Ruan $(1.7 \pm 0.4 \mathrm{~W} / \mathrm{mK})^{11}$. To compare with experiment, Teweldebrhan et al. ${ }^{15}$ measured the thermal conductivity of stacked few QLs and found that the thermal conductivity is about $1.1 \mathrm{~W} / \mathrm{mK}$, which is slightly lower than our simulation results.

Supporting on an $a-\mathrm{SiO}_{2}$ substrate leads to a $\sim 60 \%$ reduction in thermal conductivity based on our NEMD simulation results. Such a magnitude of reduction is smaller compared to some other 2D materials that supported on the same substrate. For example, Ong et al. found that the thermal conductivity of graphene is reduced by an order of magnitude once supported on an $a-\mathrm{SiO}_{2}$ substrate ${ }^{19}$. Wang et al. also reported a $78 \%$ reduction in thermal conductivity of silicene supported on $a-\mathrm{SiO}_{2}$ substrate $^{32}$. Comparing to these monolayer $2 \mathrm{D}$ materials, the substrate has less effect on the thermal conductivity of $\mathrm{Bi}_{2} \mathrm{Te}_{3} \mathrm{QL}$. This is likely due to the five-layer structure of 


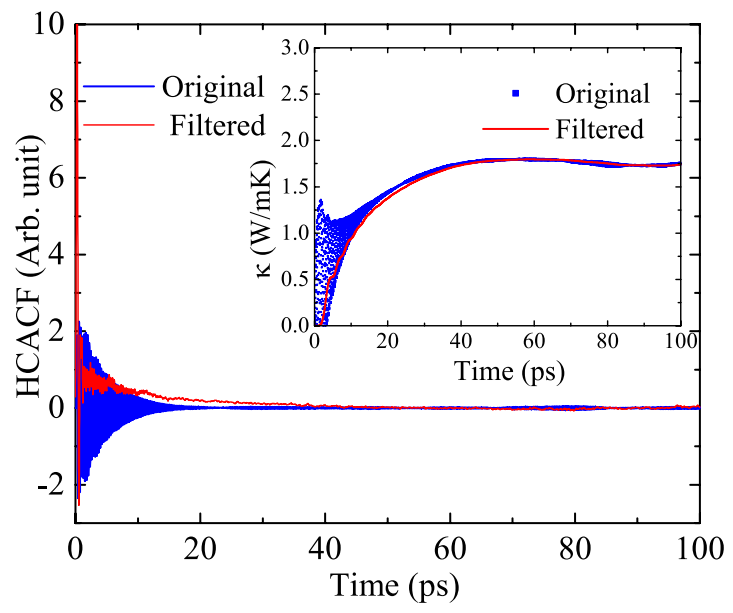

Figure 3. Typical in-plane HCACF in freestanding $\mathrm{Bi}_{2} \mathrm{Te}_{3} \mathrm{QL}$ before and after a low-pass filter (the cutoff frequency is $0.5 \mathrm{THz}$ ). Inset: The direct integral of HCACFs.

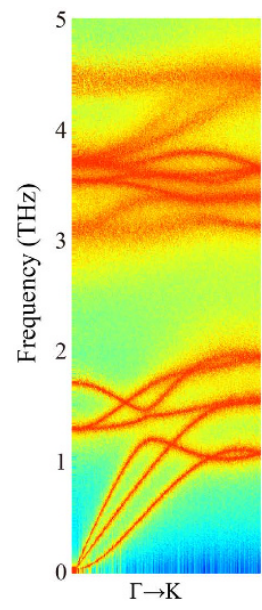

(a)

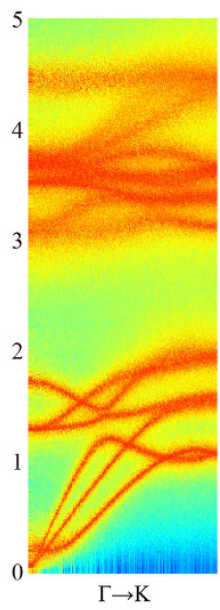

(b)

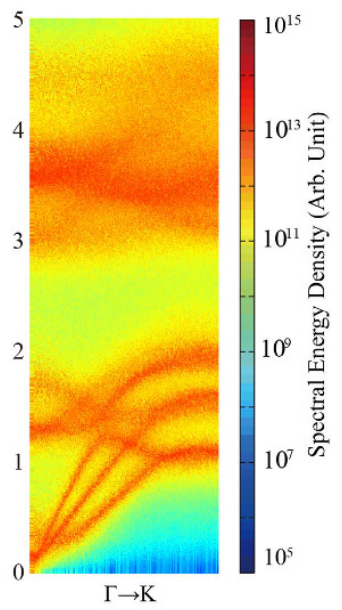

(c)

Figure 4. Spectral energy density of (a) freestanding $\mathrm{Bi}_{2} \mathrm{Te}_{3} \mathrm{QL}$, (b) supported $\mathrm{Bi}_{2} \mathrm{Te}_{3} \mathrm{QL}$, and (c) supported $\mathrm{Bi}_{2} \mathrm{Te}_{3} \mathrm{QL}$ with coupling strength 5 times of the origin one.

$\mathrm{Bi}_{2} \mathrm{Te}_{3} \mathrm{QL}$ : only the lowest layer is strongly coupled with the substrate while the other layers are relatively weakly coupled. In another work that studies the supported multilayer graphene, it was also found that the substrate suppression of the thermal conductivity is less prominent in multilayer graphene ${ }^{44}$.

Mode-wise phonon properties in $\mathrm{Bi}_{2} \mathrm{Te}_{3} \mathrm{QL}$. In order to gain a better understanding of thermal conductivity reduction in the supported $\mathrm{Bi}_{2} \mathrm{Te}_{3} \mathrm{QL}$, we perform the NMA to the freestanding and supported QL. The SED spectra of freestanding and supported QL are firstly calculated for comparison. In order to obtain a better resolution in the SED figures, a larger simulation domain $\left(131 \times 6.1 \mathrm{~nm}^{2}\right.$ in the $x$ and $y$ direction) was adopted so that $201 \mathrm{k}$-points can be extracted along $\Gamma$ to $\mathrm{K}$ direction. The total simulation time is reduced to $2 \mathrm{~ns}$. The phonon SEDs at points along the $\Gamma$ to $\mathrm{K}$ direction of freestanding and supported $\mathrm{Bi}_{2} \mathrm{Te}_{3} \mathrm{QL}$ are shown in Fig. 4(a,b). The SED spectra of supported $\mathrm{Bi}_{2} \mathrm{Te}_{3}$ with a stronger coupling strength ( 5 times of the original one) are also shown in Fig. 4(c). The magnitude of the SED is represented by different colors, as shown in Fig. 4 . The location of local maximum in the SED spectra gives the frequency of a normal mode (or phonon mode) while the broadening of the local maximum gives the phonon linewidth. Therefore, the local peaks in SED spectra give the phonon dispersion curve and the span of the peaks represents the phonon RTs.

A major difference between the supported and freestanding QL is the zone center flexural modes. For supported case, the flexural mode near the zone center is flattened and significantly broadened. A similar flattening of ZA-mode was also reported in a previous study of few layer graphene supported on $a$ - $\mathrm{SiO}_{2}$ substrate $^{45,46}$. From their studies, the coupling to the substrate has twofold effects on the supported graphene ${ }^{45,46}$. First, the dispersion of the flexural mode upward shifted from the origin. Second, the flexural mode will become linearized due to the hybridization with the surface Rayleigh mode ${ }^{45,46}$. For the supported $\mathrm{Bi}_{2} \mathrm{Te}_{3} \mathrm{QL}$, the flexural mode indeed shifted upward but the linearization is not clearly observed. The dispersion curve in supported $\mathrm{Bi}_{2} \mathrm{Te}_{3} \mathrm{QL}$ became 


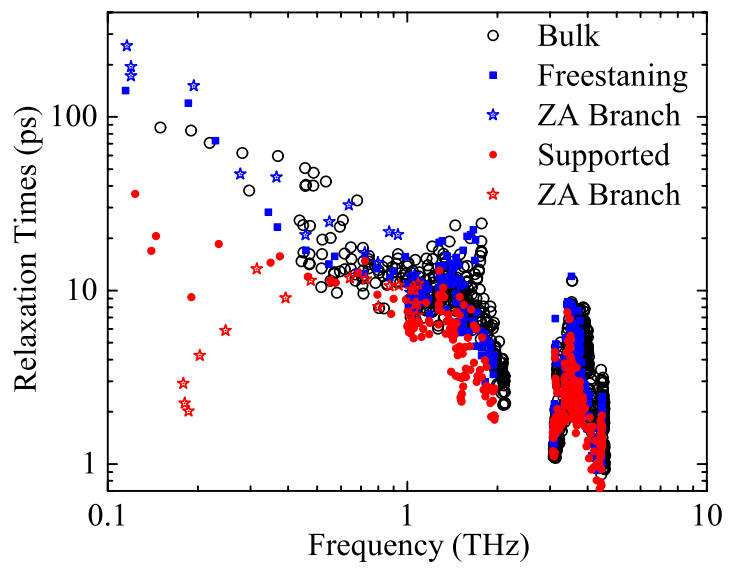

Figure 5. Phonon relaxation times in freestanding and supported $\mathrm{Bi}_{2} \mathrm{Te}_{3} \mathrm{QL}$ at $300 \mathrm{~K}$. The relaxation times in bulk $\mathrm{Bi}_{2} \mathrm{Te}_{3}$ are also shown in figure for comparison.

blurred comparing to the freestanding QL, which indicates a relatively shorter phonon RTs in the supported case. From Fig. 4(c) it can be seen that the dispersion curve became further blurred with the increasing of coupling strength. This indicates that the substrate could introduce additional phonon scattering, and the scattering strength increases with the increasing of coupling strength.

To quantitatively evaluate the effect of substrate on supported $\mathrm{Bi}_{2} \mathrm{Te}_{3} \mathrm{QL}$, we calculate the phonon RTs by fitting the SED spectra with a Lorentz function as described in Eq. (6). The results are shown in Fig. 5. Phonon RTs along $\Gamma$ to $\mathrm{K}$ point in bulk $\mathrm{Bi}_{2} \mathrm{Te}_{3}$ are also shown for comparison. It can be seen that the RTs in freestanding $\mathrm{Bi}_{2} \mathrm{Te}_{3}$ QL can be as long as $200 \mathrm{ps}$ in the low frequency region. Supporting on a substrate leads to a reduction in RTs within the frequency range. The reduction of RTs is especially strong for flexural modes, which are marked by stars in Fig. 5. The average RTs of flexural modes in freestanding QL is 46 ps, while in the supported case the average RTs is reduced to $8.5 \mathrm{ps}$. It is also reported in graphene that the flexural modes are more sensitive to the presence of substrate ${ }^{19,22}$. We believe there are two major reasons for the strong reduction of ZA mode RTs. First, the potential field from the substrate can break the selection rule for ZA phonon scattering, so that a three phonon process involving odd number of ZA modes could occur in the supported QL. This gives a larger scattering rate of ZA modes ${ }^{47}$. Second, the ZA modes in QL will hybridize with the substrate Rayleigh mode, which leads to an enhanced scattering rate between the ZA mode and the substrate mode ${ }^{46}$.

Thermal conductivity analysis. After obtaining the spectral phonon properties from the NMA and making the assumption that the first Brillouin zone is isotropic, we can express the thermal conductivity as ${ }^{32,40}$

$$
\kappa_{x}=\frac{1}{4 \pi \delta} \sum_{\nu} \int_{0}^{k_{x, \max }} c_{p h}\left(k_{x}, \nu\right) v_{g, x}^{2}\left(k_{x}, \nu\right) \tau\left(k_{x}, \nu\right) k_{x} \mathrm{~d} k_{x},
$$

where $\kappa_{x}$ is the thermal conductivity in $x$ direction, $\delta$ is the thickness of $\mathrm{Bi}_{2} \mathrm{Te}_{3} \mathrm{QL}, \nu$ represents different polarization, $c_{p h}\left(k_{x}, \nu\right)$ is the phonon specific heat of phonon mode $\left(k_{x}, \nu\right), v_{g, x}\left(k_{x}, \nu\right)$ is the phonon group velocity in $x$ direction calculated from harmonic lattice dynamics, and $\tau\left(k_{x}, \nu\right)$ is the phonon relaxation time.

The overall thermal conductivities of freestanding and supported $\mathrm{Bi}_{2} \mathrm{Te}_{3}$ QL predicted by NMA are 2.1 and $1.0 \mathrm{~W} / \mathrm{mK}$, which agree relativity well with that calculated from NEMD and Green-Kubo method. From the NMA calculation, the substrate leads to a $\sim 50 \%$ reduction in thermal conductivity, which is also similar to the NEMD results.

We further build the accumulated thermal conductivity with respect to MFP, which can be expressed as

$$
\kappa_{\text {accum }}\left(\lambda^{*}\right)=\sum_{\nu} \int_{0}^{\lambda^{*}} \frac{1}{3} c_{\lambda}(\lambda) v_{g}(\lambda) \lambda \mathrm{d} \lambda,
$$

where $\lambda^{*}$ is the cutoff MFP, $c_{\lambda}(\lambda)$ is the volumetric heat capacity per unit MFP, and the sum is over all polarizations. The phonon MFP $\lambda$ and relaxation time $\tau$ has the relationship $\lambda=v_{g} \tau$. In practice, we used a discrete summation over phonon modes with MFP less than $\lambda^{*}$ to calculate the integral in Eq. (8). The accumulated thermal conductivities are shown in Fig. 6. Comparing the accumulated thermal conductivity of freestanding and supported $\mathrm{Bi}_{2} \mathrm{Te}_{3} \mathrm{QL}$, it can be seen that the reduction in thermal conductivity of supported QL is mainly due to the suppression of long MFP phonon modes. Below $20 \mathrm{~nm}$, the accumulated thermal conductivity of freestanding and supported $\mathrm{Bi}_{2} \mathrm{Te}_{3} \mathrm{QL}$ are almost the same, while the thermal conductivity contributed by phonon with MFPs larger than $20 \mathrm{~nm}$ is $1.2 \mathrm{~W} / \mathrm{mK}$ in freestanding $\mathrm{Bi}_{2} \mathrm{Te}_{3} \mathrm{QL}$ and $0.2 \mathrm{~W} / \mathrm{mK}$ in supported $\mathrm{Bi}_{2} \mathrm{Te}_{3} \mathrm{QL}$. We can also see that half of the heat in freestanding $\mathrm{Bi}_{2} \mathrm{Te}_{3} \mathrm{QL}$ is carried by phonons with MFPs larger than $52.4 \mathrm{~nm}$, while in supported QL this value is reduced to $24.2 \mathrm{~nm}$. The accumulated thermal conductivity as a function of MFP for bulk $\mathrm{Bi}_{2} \mathrm{Te}_{3}$ is also shown in Fig. 6 for comparison. It has been previously demonstrated by EMD simulations that $\mathrm{Bi}_{2} \mathrm{Te}_{3} \mathrm{QL}$ has a larger thermal conductivity than bulk $\mathrm{Bi}_{2} \mathrm{Te}_{3}{ }^{11}$. From the accumulated thermal conductivity 


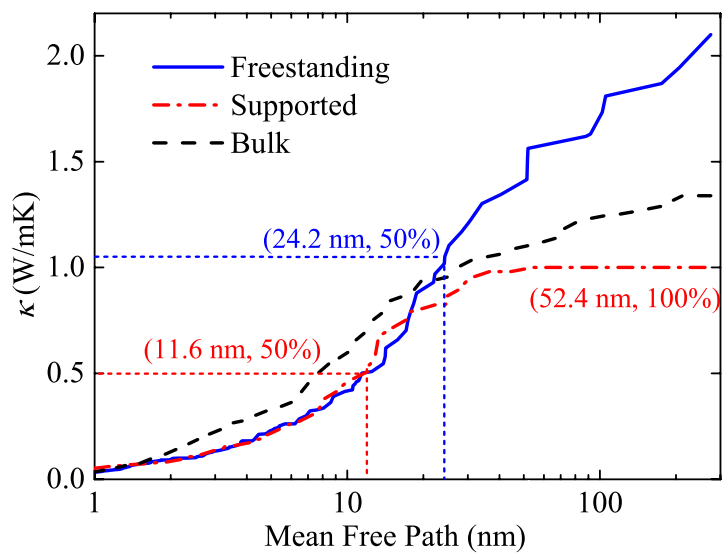

Figure 6. Accumulated thermal conductivities in freestanding and supported $\mathrm{Bi}_{2} \mathrm{Te}_{3} \mathrm{QL}$ with respect to phonon MFP. The accumulated thermal conductivity in bulk $\mathrm{Bi}_{2} \mathrm{Te}_{3}$ is also shown figure for comparison.

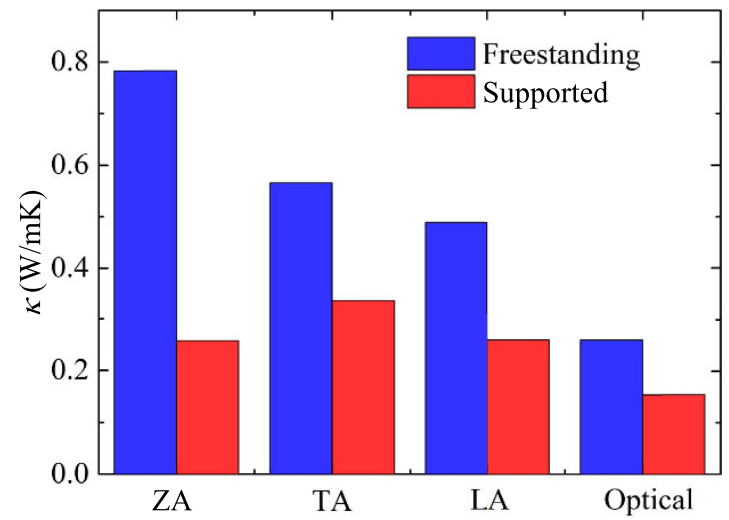

Figure 7. Thermal conductivity of freestanding and supported $\mathrm{Bi}_{2} \mathrm{Te}_{3} \mathrm{QL}$ contributed from different phonon branches.

function, it can be seen that such an enhancement is mainly contributed by the phonons with MFPs longer than $25 \mathrm{~nm}$ (phonon modes near zone center) in $\mathrm{Bi}_{2} \mathrm{Te}_{3} \mathrm{QL}$.

The thermal conductivity contributed from different branches, namely out-of-plane (ZA), longitudinal acoustic (LA), transverse acoustic (TA), and optical branches are also analyzed and the results are shown in Fig. 7. For freestanding $\mathrm{Bi}_{2} \mathrm{Te}_{3} \mathrm{QL}$, the $\mathrm{ZA}$ branch contributes most of the thermal conductivity (37\%) and the contribution from all three acoustic branches (ZA, TA and LA branch) is $88 \%$. The contribution from optical branch is very small. It should be noted that the large contribution from ZA mode to the heat transfer is not unusual for $2 \mathrm{D}$ materials. For materials with reflectional symmetry in the $x y$ plane (e.g. graphene), due to the unique selection rule for phonon scattering, the ZA modes tend to scatter less with other phonon modes ${ }^{47}$. Placing on the substrate will suppress the thermal conductivity of all branches, while the suppression in ZA branch is the most significant. From the relaxation times in Fig. 5, it can be seen that this is mainly due to the largely reduced in relaxation time of ZA modes.

Effect of substrate atomic mass and interaction strength. When a 2D material is coupled with another material, for example, supported by a substrate, the detailed interaction and adhesion energy may be quite different, depending on the material types, the interfacial geometry, and the surface polarity of substrate ${ }^{48,49}$. It is also reported that the thermal conductivity of supported materials could be quite different depending on the coupling strength and mass ratio to the substrate ${ }^{20}$. In fact this feature could be utilized to tune the thermal conductivity of supported materials ${ }^{20,23}$. To explore the effects of different substrate, we generalize our substrate to a virtual one with different atomic masses and coupling strengths with the supported QL. The NMA in combination with Eq. (7) is used to calculate the thermal conductivity of supported $\mathrm{Bi}_{2} \mathrm{Te}_{3} \mathrm{QL}$ on different virtual substrates. The coupling strength is tuned by scaling the potential depth $\varepsilon$ in the LJ potential for all atom-pairs between the substrate and the QL. The results are shown as the inset of Fig. 8. We can see that increasing the coupling strength tends to further reduce the thermal conductivity of $\mathrm{Bi}_{2} \mathrm{Te}_{3} \mathrm{QL}$. Doubling the interaction strength could reduce the thermal conductivity to $0.63 \mathrm{~W} / \mathrm{mK}$. Further increasing the coupling strength leads to a further reduction in thermal conductivity. It should be noted that in the case of 10 times the coupling strength, the adhesion energy between substrate and QL becomes $0.91 \mathrm{~J} / \mathrm{m}^{2}$, while the adhesion energy between the atomic layers in QL is $9.8 \mathrm{~J} / \mathrm{m}^{2}$. In this case the QL and the substrate are relatively strongly coupled and it can be inferred from Fig. 4 that the zone 


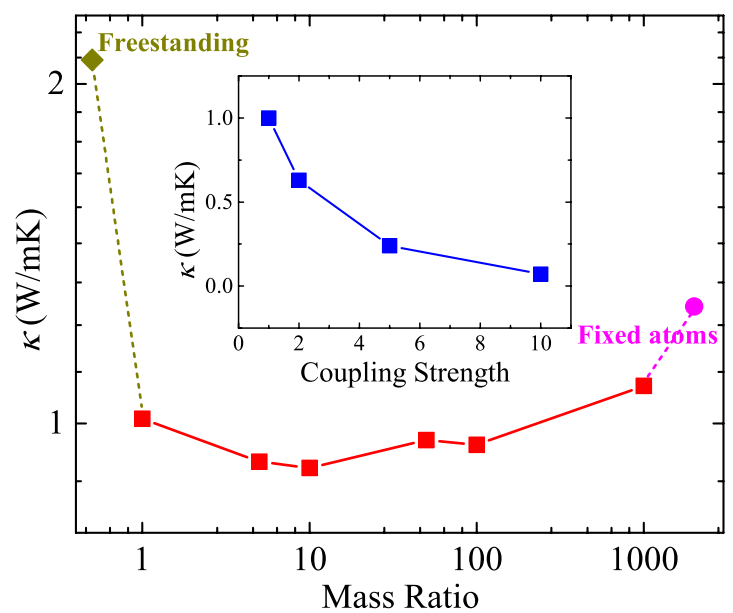

Figure 8. Thermal conductivity of supported $\mathrm{Bi}_{2} \mathrm{Te}_{3} \mathrm{QL}$ with different substrate atomic masses and different coupling strengths.

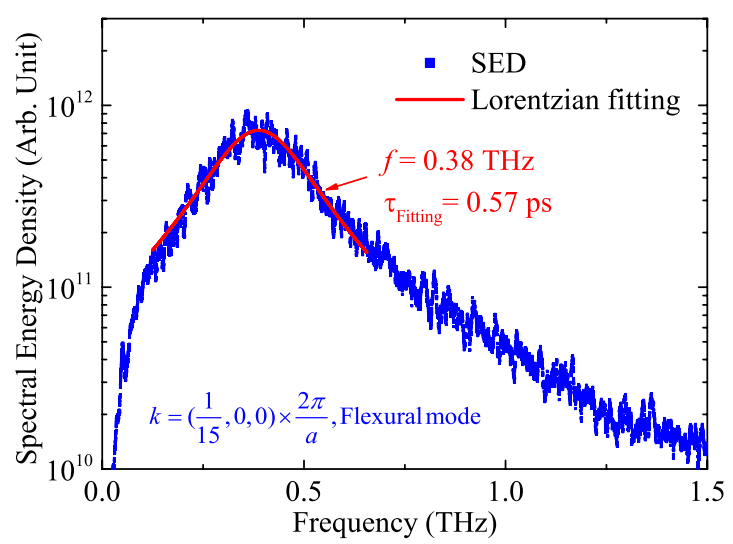

Figure 9. Typical SED profile of a zone center flexural mode for the supported QL.

center flexural branch is flattened due to the existence of substrate. This means that the phonon frequencies and eigenvectors of the supported QL are changed due to the perturbation from the substrate. In such case, whether the eigenvectors for the freestanding QL are applicable for the perturbed system remains unclear ${ }^{50,51}$. To demonstrate the applicability of the unperturbed eigenvectors to the perturbed system, we check the SED profiles of zone center flexural modes with the 10 times coupling strength and plot a typical spectrum (flexural mode with wavevector $k=(1 / 15$, $0,0) \times 2 \pi / a)$ in Fig. 9 . We can clearly see a single peak in the SED profile with no significant disruption, indicating that the eigenvectors of the freestanding QL are robust enough to resolve the phonon modes in the supported QL. This further confirms the accuracy of our NMA calculation on the RTs and thermal conductivity for the supported case.

We also scale the atomic mass of all atoms in the substrate by a factor (mass ratio) to explore the effects of substrate atomic mass on the thermal transport in supported materials. The relationship between thermal conductivity and atomic mass ratio of substrate is shown in Fig. 8. We can see that with the increasing of the atomic mass, thermal conductivity of supported $\mathrm{Bi}_{2} \mathrm{Te}_{3}$ QL does not change much, except for the cases when the mass ratio is 1000 or when the atoms fixed. When the atomic mass is 1000 , the substrate becomes almost immovable and the direct scattering process between phonon in $\mathrm{Bi}_{2} \mathrm{Te}_{3} \mathrm{QL}$ and phonons in substrate is largely suppressed ${ }^{45}$. The presence of substrate can affect the thermal transport in supported materials from two different aspects: the static potential at the interface introduced by the substrate and the dynamics of the substrate itself ${ }^{52}$. These two effects can be decoupled through comparing the thermal conductivity of QL under different environment: freestanding, supported, and supported on a substrate with fixed atoms. For the case of substrate with fixed atoms, the reduction of thermal conductivity is mainly due to the static potential at the interface. From Fig. 8, it can be seen that this effect leads to a $40 \%$ reduction in thermal conductivity. Allowing the atoms in the substrate to move will introduce additional scattering with substrate phonons, which leads to an additional $13 \%$ reduction in thermal conductivity.

Summary. To summarize, we have systematically investigated the mode-resolved phonon transport properties of freestanding and supported $\mathrm{Bi}_{2} \mathrm{Te}_{3}$ QL by the MD-based NMA methods. The thermal conductivities of freestanding and supported $\mathrm{Bi}_{2} \mathrm{Te}_{3} \mathrm{QL}$ are predicted to be 2.1 and $1.0 \mathrm{~W} / \mathrm{mK}$, respectively. These values are 
consistent with the predictions from NEMD and Green-Kubo methods. It is shown that the phonon RTs in the $\mathrm{Bi}_{2} \mathrm{Te}_{3} \mathrm{QL}$ range from 1 to $200 \mathrm{ps}$. Some low frequency phonons have evidently larger relaxation time than that in the bulk $\mathrm{Bi}_{2} \mathrm{Te}_{3}$, which are responsible for the larger thermal conductivity of the QL comparing to bulk phase. The accumulated thermal conductivity is also constructed, which shows that about $60 \%$ of the heat is carried by phonons with MFP longer than $20 \mathrm{~nm}$ in the QL. The low thermal conductivity of supported QL is mainly attributed to the reduced phonon RTs of all the frequency range, and most significantly for ZA phonon modes. By further varying the coupling strength with substrate and the atomic mass of substrate, we conclude that interfacial scattering is the dominating factor that leads to a reduction of about $40 \%$ of the thermal conductivity of $\mathrm{Bi}_{2} \mathrm{Te}_{3} \mathrm{QL}$. Further increasing the coupling strength can significantly suppress the thermal conductivity in $\mathrm{Bi}_{2} \mathrm{Te}_{3} \mathrm{QL}$. Scattering with the substrate phonons can reduce the thermal conductivity by about $13 \%$. Moderate variation of the atomic mass of the substrate has a relatively small effect on the thermal conductivity of the supported QL.

\section{References}

1. Poudel, B. et al. High-thermoelectric performance of nanostructured bismuth antimony telluride bulk alloys. Science 320, 634-638 (2008).

2. Venkatasubramanian, R., Siivola, E., Colpitts, T. \& O'quinn, B. Thin-film thermoelectric devices with high room-temperature figures of merit. Nature 413, 597-602 (2001).

3. Tang, X. et al. Preparation and thermoelectric transport properties of high-performance p-type $\mathrm{Bi}_{2} \mathrm{Te}_{3}$ with layered nanostructure. Appl. Phys. Lett. 90, 012102 (2007).

4. Zhao, X. B. et al. Bismuth telluride nanotubes and the effects on the thermoelectric properties of nanotube-containing nanocomposites. Appl. Phys. Lett. 86, 062111 (2005).

5. Cao, Y. Q., Zhao, X. B., Zhu, T. J., Zhang, X. B. \& Tu, J. P. Syntheses and thermoelectric properties of $\mathrm{Bi}_{2} \mathrm{Te}_{2} \mathrm{Sb}_{2} \mathrm{Te}_{3}$ bulk nanocomposites with laminated nanostructure. Appl. Phys. Lett. 92, 143106 (2008).

6. Hicks, L. D. \& Dresselhaus, M. S. Thermoelectric figure of merit of a one-dimensional conductor. Phys. Rev. B 47, 16631 (1993).

7. Balandin, A. \& Wang, K. L. Significant decrease of the lattice thermal conductivity due to phonon confinement in a free-standing semiconductor quantum well. Phys. Rev. B 58, 1544 (1998).

8. Dresselhaus, M. S. et al. New directions for low-dimensional thermoelectric materials. Adv. Mater. 19, 1043-1053 (2007).

9. Teweldebrhan, D., Goyal, V., Rahman, M. \& Balandin, A. A. Atomically-thin crystalline films and ribbons of bismuth telluride. Appl. Phys. Lett. 96, 053107 (2010).

10. Zhang, J. et al. Enhanced thermoelectric performance of a quintuple layer of $\mathrm{Bi}_{2} \mathrm{Te}_{3}$. J. Appl. Phys. 116, 023706 (2014).

11. Qiu, B. \& Ruan, X. Thermal conductivity prediction and analysis of few-quintuple $\mathrm{Bi}_{2} \mathrm{Te}_{3}$ thin films: A molecular dynamics study. Appl. Phys. Lett. 97, 183107 (2010).

12. Park, K. H., Mohamed, M., Aksamija, Z. \& Ravaioli, U. Phonon scattering due to van der Waals forces in the lattice thermal conductivity of $\mathrm{Bi}_{2} \mathrm{Te}_{3}$ thin films. J. Appl. Phys. 117, 015103 (2015).

13. Pernot, G. et al. Precise control of thermal conductivity at the nanoscale through individual phonon-scattering barriers. Nature Mater. 9, 491-495 (2010).

14. Hu, Y., Zeng, L., Minnich, A. J., Dresselhaus, M. S. \& Chen, G. Spectral mapping of thermal conductivity through nanoscale ballistic transport. Nature Nanotech. 10, 701-706 (2015).

15. Teweldebrhan, D., Goyal, V. \& Balandin, A. A. Exfoliation and characterization of bismuth telluride atomic quintuples and quasitwo-dimensional crystals. Nano Lett. 10, 1209-1218 (2010).

16. Jia, L., Ma, W. \& Zhang, X. Ultrafast carrier dynamics in polycrystalline bismuth telluride nanofilm. Appl. Phys. Lett. 104, 241911 (2014).

17. Liao, Q., Liu, Z., Liu, W., Deng, C. \& Yang, N. Extremely high thermal conductivity of aligned carbon nanotube-polyethylene composites. Sci. Rep. 5, 16543 (2015).

18. Seol, J. H. et al. Two-dimensional phonon transport in supported graphene. Science 328, 213-216 (2010).

19. Ong, Z.-Y. \& Pop, E. Effect of substrate modes on thermal transport in supported graphene. Phys. Rev. B 84, 075471 (2011).

20. Guo, Z.-X., Zhang, D. \& Gong, X.-G. Manipulating thermal conductivity through substrate coupling. Phys. Rev. B 84, 075470 (2011).

21. Yue, Y., Zhang, J., Tang, X., Xu, S. \& Wang, X. Thermal transport across atomic-layer material interfaces. Nanotechnol. Rev. 4, 533 (2015).

22. Qiu, B. \& Ruan, X. Reduction of spectral phonon relaxation times from suspended to supported graphene. Appl. Phys. Lett. 100, 193101 (2012).

23. Zhang, X., Bao, H. \& Hu, M. Bilateral substrate effect on the thermal conductivity of two-dimensional silicon. Nanoscale 7, 6014-6022 (2015).

24. Plimpton, S. Fast parallel algorithms for short-range molecular dynamics. J. Comput. Phys. 117, 1 (1995).

25. Hellman, O. \& Broido, D. A. Phonon thermal transport in $\mathrm{Bi}_{2} \mathrm{Te}_{3}$ from first principles. Phys. Rev. B 90, 134309 (2014).

26. Feng, T. \& Ruan, X. Prediction of spectral phonon mean free path and thermal conductivity with applications to thermoelectrics and thermal management: a review. J. Nanomater. 2014, e206370 (2014).

27. Xie, H., Hu, M. \& Bao, H. Thermal conductivity of silicene from first-principles. Appl. Phys. Lett. 104, 131906 (2014).

28. Qiu, B. \& Ruan, X. Molecular dynamics simulations of lattice thermal conductivity of bismuth telluride using two-body interatomic potentials. Phys. Rev. B 80, 165203 (2009).

29. Munetoh, S., Motooka, T., Moriguchi, K. \& Shintani, A. Interatomic potential for Si-O systems using Tersoff parameterization. Comput. Mater. Sci. 39, 334 (2007).

30. Shao, C. \& Bao, H. A molecular dynamics investigation of heat transfer across a disordered thin film. Int. J. Heat Mass Transfer 85, 33-40 (2015).

31. Ong, Z.-Y. \& Pop, E. Molecular dynamics simulation of thermal boundary conductance between carbon nanotubes and $\mathrm{SiO}_{2}$. Phys. Rev. B 81, 155408 (2010).

32. Wang, Z., Feng, T. \& Ruan, X. Thermal conductivity and spectral phonon properties of freestanding and supported silicene. J. Appl. Phys. 117, 084317 (2015).

33. Rappé, A. K., Casewit, C. J., Colwell, K. S., Goddard Iii, W. A. \& Skiff, W. M. UFF, a full periodic table force field for molecular mechanics and molecular dynamics simulations. J. Am. Chem. Soc. 114, 10024-10035 (1992).

34. Bao, H., Shao, C., Luo, S. \& Hu, M. Enhancement of interfacial thermal transport by carbon nanotube-graphene junction. J. Appl. Phys. 115, 053524 (2014).

35. McGaughey, A. Predicting phonon properties from equilibrium molecular dynamics simulations. Ann. Rev. Heat Transfer 17, 49-87 (2014).

36. Huang, B.-L. \& Kaviany, M. Ab initio and molecular dynamics predictions for electron and phonon transport in bismuth telluride. Phys. Rev. B 77, 125209 (2008).

37. Dove, M. T. Introduction to lattice dynamics. 93-94 (Cambridge university press, 1993). 
38. Bao, H., Qiu, B., Zhang, Y. \& Ruan, X. A first-principles molecular dynamics approach for predicting optical phonon lifetimes and far-infrared reflectance of polar materials. J. Quant. Spectrosc. Radiat. Transfer 113, 1683-1688 (2012).

39. Ladd, A. J. C., Moran, B. \& Hoover, W. G. Lattice thermal conductivity: A comparison of molecular dynamics and anharmonic lattice dynamics. Phys. Rev. B 34, 5058-5064 (1986).

40. Wang, Y., Qiu, B., McGaughey, A. J., Ruan, X. \& Xu, X. Mode-wise thermal conductivity of bismuth telluride. J. Heat Transfer 135, $091102(2013)$.

41. Qiu, B., Bao, H., Zhang, G., Wu, Y. \& Ruan, X. Molecular dynamics simulations of lattice thermal conductivity and spectral phonon mean free path of PbTe: Bulk and nanostructures. Comput. Mater. Sci. 53, 278-285 (2012).

42. Gale, J. D. GULP: A computer program for the symmetry-adapted simulation of solids. J. Chem. Soc., Faraday Trans. 93, 629-637 (1997).

43. Sellan, D. P., Landry, E. S. \& Turney, J. E. Size effects in molecular dynamics thermal conductivity predictions. Phys. Rev. B 81, 214305 (2010).

44. Chen, J., Zhang, G. \& Li, B. Substrate coupling suppresses size dependence of thermal conductivity in supported graphene. Nanoscale 5, 532-536 (2013).

45. Ni, Y., Kosevich, Y. A., Xiong, S., Chalopin, Y. \& Volz, S. Substrate-induced cross-plane thermal propagative modes in few-layer graphene. Phys. Rev. B 89, 205413 (2014).

46. Amorim, B. \& Guinea, F. Flexural mode of graphene on a substrate. Phys. Rev. B 88, 115418 (2013).

47. Lindsay, L., Broido, D. A. \& Mingo, N. Flexural phonons and thermal transport in graphene. Phys. Rev. B 82, 115427 (2010).

48. Kang, Y.-J., Kang, J. \& Chang, K. J. Electronic structure of graphene and doping effect on $\mathrm{SiO}_{2}$. Phys. Rev. B 78, 115404 (2008)

49. Sachs, B., Wehling, T. O., Katsnelson, M. I. \& Lichtenstein, A. I. Adhesion and electronic structure of graphene on hexagonal boron nitride substrates. Phys. Rev. B 84, 195414 (2011)

50. Huberman, S., Larkin, J., McGaughey, A. \& Amon, C. Disruption of superlattice phonons by interfacial mixing. Phys. Rev. B 88, 155311 (2013).

51. Feng, T., Qiu, B. \& Ruan, X. Anharmonicity and necessity of phonon eigenvectors in the phonon normal mode analysis. J. Appl. Phys. 117, 195102 (2015).

52. Ong, Z.-Y., Pop, E. \& Shiomi, J. Reduction of phonon lifetimes and thermal conductivity of a carbon nanotube on amorphous silica. Phys. Rev. B 84, 165418 (2011).

\section{Acknowledgements}

This work is supported by the National Natural Science Foundation of China (No. 51306111) and Shanghai Municipal Natural Science Foundation (No. 13ZR1456000). The computational support from Center for High Performance Computing at Shanghai Jiao Tong University $(\pi)$ is also gratefully appreciated. C.S. would like to thank Dr. Bo Qiu for sharing the NMA code and Tianli Feng for fruitful discussions.

\section{Author Contributions}

C.S. performed the simulations under the supervising of H.B. C.S. and H.B. analyzed the data. C.S. drafted the manuscript. H.B. reviewed and edited the manuscript.

\section{Additional Information}

Competing financial interests: The authors declare no competing financial interests.

How to cite this article: Shao, C. and Bao, H. Thermal transport in bismuth telluride quintuple layer: moderesolved phonon properties and substrate effects. Sci. Rep. 6, 27492; doi: 10.1038/srep27492 (2016).

(c) (i) This work is licensed under a Creative Commons Attribution 4.0 International License. The images or other third party material in this article are included in the article's Creative Commons license, unless indicated otherwise in the credit line; if the material is not included under the Creative Commons license, users will need to obtain permission from the license holder to reproduce the material. To view a copy of this license, visit http://creativecommons.org/licenses/by/4.0/ 